\title{
Generalizations of weighted means and OWA operators by using unimodal weighting vectors
}

\author{
Bonifacio Llamazares
}

\begin{abstract}
Weighted means and OWA operators are two families of functions well known in the literature. Given that both are specific cases of the Choquet integral, several procedures for constructing capacities that generalize simultaneously those of the weighted means and the OWA operators have been suggested in recent years. In this paper we propose two methods that allow us to address the previous issue and that provide us with a wide variety of capacities when the weighting vector associated with the OWA operator is unimodal.
\end{abstract}

Index Terms-Weighted means, OWA operators, unimodal weighting vectors, SUOWA operators, Semi-SUOWA operators, the Crescent Method, Choquet integral.

\section{INTRODUCTION}

Weighted means and the ordered weighted averaging (OWA) operators [1] are two families of functions widely used in the field of aggregation operators. Due to their importance, in recent years have appeared in the literature several procedures to construct operators that allow to generalize simultaneously both families of functions (see, for instance, [2]-[5], and [6], [7] for an analysis of some of them). The approach followed by most authors is to consider operators parametrized by two weighting vectors, $\boldsymbol{p}$ for the weighted mean and $\boldsymbol{w}$ for the OWA operator, so that we can recover the weighted mean when $\boldsymbol{w}=(1 / n, \ldots, 1 / n)$ and the OWA operator when $\boldsymbol{p}=$ $(1 / n, \ldots, 1 / n)$.

Two of the most interesting generalizations are the weighted ordered weighted averaging (WOWA) operators [2] and the semiuninorm-based ordered weighted averaging (SUOWA) operators [3] because both can be expressed through Choquet integrals with respect to known normalized capacities (it is worth noting that the operators proposed in [4] are also Choquet integrals but their capacities are unknown). SUOWA operators have some important advantages over the other approaches proposed in the literature (see [8] for a behavioral analysis of WOWA and SUOWA operators): On the one hand, some indices such as the orness degree [1], [9], the Shapley value [10], the veto and favor indices [9], and the $k$ conjunctiveness and $k$-disjunctiveness indices [11] are given through closed-form expressions for some specific cases of SUOWA operators [12]. On the other hand, it is possible to obtain SUOWA operators ranging between two order statistics, which is not feasible for other families of functions [7].

It is also important to note that some families of functions recently introduced in the literature are closely related to SUOWA operators. For instance:

B. Llamazares is with the Departamento de Economía Aplicada and the Instituto de Matemáticas (IMUVA), Universidad de Valladolid, 47011 Valladolid, Spain, e-mail: boni@eco.uva.es.
1) The games obtained with the Crescent Method [13] can be expressed as a two-piecewise function where the first piece coincides with a game associated with a SUOWA operator and the second piece is the dual of a game also obtained in the context of SUOWA operators [14].

2) A natural generalization of the Winsorized mean [15], [16], called Winsorized weighted mean [17], is a specific case of SUOWA operators.

3) The solution of the convex optimization problem proposed in [18] is also a specific case of SUOWA operators [8], [12], [19].

Although SUOWA operators possess interesting properties, their main weakness is that, sometimes, the construction of the capacities is not straightforward given that it is necessary to calculate the monotonic cover [20], [21] of certain games, which are obtained by using semiuninorms [22] with neutral element $1 / n$ and the values of the capacities associated with the weighted means and the OWA operators. However, we can directly get normalized capacities when the weighting vector $\boldsymbol{w}$ is unimodal and a specific semiuninorm, $U_{\min }^{\max }$, is used [23].

Given that unimodal weighting vectors embrace some of the most outstanding weighting vectors used in the framework of OWA operators (nonincreasing, nondecreasing, centered weighting vectors, etc.), the aim of this paper is to present two procedures that allows us to obtain normalized capacities (without the need to use the monotonic cover) when the weighting vector $\boldsymbol{w}$ is unimodal. In both methods the capacities are constructed by using the function $H(x, y)=$ $h^{(-1)}(h(x)+h(y)-h(1 / n))$, where $h$ has to fulfill certain conditions and $h^{(-1)}$ is the pseudo-inverse of $h$. In the first method, we introduce new families of semiuninorms by means of the functions $H$, so the resulting functions are SUOWA operators. In the second one, we generalize the equivalent representation of the Crescent Method given in [14], and the Choquet integrals associated with the resulting capacities will be called Semi-SUOWA operators. Hence, as an immediate consequence, we get that the Crescent Method allows to obtain normalized capacities when the weighting vector $\boldsymbol{w}$ is unimodal.

The remainder of the paper is organized as follows. Section II recalls basic concepts of SUOWA operators and pseudoinverse functions. In Section III we show some properties of unimodal weighting vectors. Sections IV and V are dedicated to introduce the proposed methods and prove that both allow to obtain normalized capacities when $\boldsymbol{w}$ is unimodal. In Section VI we give a wide variety of functions $H$, which are used in both procedures. Finally, some concluding remarks are provided in Section VII. 


\section{PRELIMINARIES}

The following notation will be used throughout the paper: $N$ denotes the set $\{1, \ldots, n\},|A|$ and $A^{\mathrm{c}}$ denote, respectively, the cardinality and the complement of a subset $A$ of $N$, vectors are denoted in bold, $\boldsymbol{\eta}$ is the vector $(1 / n, \ldots, 1 / n) \in \mathbb{R}^{n}$, and given $\boldsymbol{x} \in \mathbb{R}^{n},[\cdot]$ and $(\cdot)$ denote permutations such that $x_{[1]} \geq \cdots \geq x_{[n]}$ and $x_{(1)} \leq \cdots \leq x_{(n)}$.

SUOWA operators are specific cases of Choquet integrals [24]-[26], which are constructed by using normalized capacities (see [27] for an interesting study on the constructions of normalized capacities by means of aggregation and implication functions). A game $v$ on $N$ is a set function, $v: 2^{N} \longrightarrow \mathbb{R}$ satisfying $v(\varnothing)=0$. A monotonic game is called a capacity, and a capacity $\mu$ is normalized if $\mu(N)=1$.

The monotonic cover [20], [21] of a game $v$ is the set function $\widehat{v}$ given by

$$
\widehat{v}(A)=\max _{B \subseteq A} v(B) .
$$

By construction, $\widehat{v}$ is a capacity, and $\widehat{v}=v$ when $v$ is a capacity. Moreover, $\widehat{v}$ is a normalized capacity when $v(N)=$ 1 and $v(A) \leq 1$ for all $A \subseteq N$.

The dual of a game $v$ is the game defined by

$$
\bar{v}(A)=1-v\left(A^{\mathrm{c}}\right) \quad(A \subseteq N) .
$$

It is easy to check that the dual of a normalized capacity is also a normalized capacity.

The Choquet integral with respect to a normalized capacity $\mu$ is the function $\mathcal{C}_{\mu}: \mathbb{R}^{n} \longrightarrow \mathbb{R}$ given by

$$
\mathcal{C}_{\mu}(\boldsymbol{x})=\sum_{i=1}^{n} \mu\left(A_{[i]}\right)\left(x_{[i]}-x_{[i+1]}\right),
$$

where $A_{[i]}=\{[1], \ldots,[i]\}$, and we adopt the convention that $x_{[n+1]}=0$. Alternatively, the Choquet integral can be also expressed as

$$
\mathcal{C}_{\mu}(\boldsymbol{x})=\sum_{i=1}^{n}\left(\mu\left(A_{[i]}\right)-\mu\left(A_{[i-1]}\right)\right) x_{[i]},
$$

where we use the convention $A_{[0]}=\varnothing$.

Two well-known specific cases of Choquet integrals are the weighted means and the OWA operators, which are defined through weighting vectors; that is, nonnegative vectors whose components sum to 1 . The weighted mean $M_{p}$ associated with a weighting vector $\boldsymbol{p}$ is the Choquet integral with respect to the normalized capacity $\mu_{\boldsymbol{p}}(A)=\sum_{i \in A} p_{i}$; that is,

$$
M_{\boldsymbol{p}}(\boldsymbol{x})=\sum_{i=1}^{n} p_{i} x_{i} .
$$

The OWA operator $O_{\boldsymbol{w}}$ associated with a weighting vector $\boldsymbol{w}$ is the Choquet integral with respect to the normalized capacity $\mu_{|\boldsymbol{w}|}(A)=\sum_{i=1}^{|A|} w_{i}$; that is,

$$
O_{\boldsymbol{w}}(\boldsymbol{x})=\sum_{i=1}^{n} w_{i} x_{[i]} .
$$

Notice that OWA operators are convex combinations of order statistics. Moreover, the dual of $\mu_{|\boldsymbol{w}|}$ (that for the sake of simplicity we will denote by $\bar{\mu}_{|\boldsymbol{w}|}$ instead of $\overline{\mu_{|\boldsymbol{w}|}}$ ) is given by $\mu_{|\overline{\boldsymbol{w}}|}$, where $\overline{\boldsymbol{w}}$ is the dual of $\boldsymbol{w}$; that is, $\overline{\boldsymbol{w}}=$ $\left(w_{n}, w_{n-1}, \ldots, w_{1}\right)$ (equivalently, $\left.\bar{w}_{i}=w_{n+1-i}\right)$.

Semiuninorms [22] play a fundamental role in the definition of SUOWA operators. A semiuninorm is a nondecreasing binary operation $U:[0,1]^{2} \longrightarrow[0,1]$ that has a neutral element. The set of semiuninorms with $1 / n$ as neutral element is denoted by $\mathcal{U}^{1 / n}$, and the semiuninorms used in the definition of SUOWA operators have to belong to the following subset (see [28] for a graphical representation of some of them):

$$
\widetilde{\mathcal{U}}^{1 / n}=\left\{U \in \mathcal{U}^{1 / n} \mid U(1 / k, 1 / k) \leq 1 / k \text { for all } k \in N\right\} \text {. }
$$

Notice that semiuninorms are generalizations of uninorms [29] (where the commutativity and associativity properties are ruled out), which have been widely studied in the literature [30]. Next we recall the definition of SUOWA operators [3].

Definition 1: Let $\boldsymbol{p}$ and $\boldsymbol{w}$ be two weighting vectors and let $U \in \widetilde{\mathcal{U}}^{1 / n}$.

1) The game associated with $\boldsymbol{p}, \boldsymbol{w}$ and $U$ is the set function $v_{\boldsymbol{p}, \boldsymbol{w}}^{U}: 2^{N} \longrightarrow \mathbb{R}$ defined by

$$
\begin{aligned}
v_{\boldsymbol{p}, \boldsymbol{w}}^{U}(A) & =|A| U\left(\frac{\mu_{\boldsymbol{p}}(A)}{|A|}, \frac{\mu_{|\boldsymbol{w}|}(A)}{|A|}\right) \\
& =|A| U\left(\frac{\sum_{i \in A} p_{i}}{|A|}, \frac{\sum_{i=1}^{|A|} w_{i}}{|A|}\right),
\end{aligned}
$$

if $A \neq \varnothing$, and $v_{\boldsymbol{p}, \boldsymbol{w}}^{U}(\varnothing)=0$.

2) $\widehat{v}_{\boldsymbol{p}, \boldsymbol{w}}^{U}$, the monotonic cover of the game $v_{\boldsymbol{p}, \boldsymbol{w}}^{U}$, will be called the capacity associated with $\boldsymbol{p}, \boldsymbol{w}$ and $U$.

3) The SUOWA operator associated with $\boldsymbol{p}, \boldsymbol{w}$ and $U$ is the Choquet integral with respect to the capacity $\widehat{v}_{\boldsymbol{p}, \boldsymbol{w}}^{U}$.

Note that, by construction, SUOWA operators allow us to recover the weighted mean $M_{\boldsymbol{p}}$ when $\boldsymbol{w}=\boldsymbol{\eta}$ and the OWA operator $O_{\boldsymbol{w}}$ when $\boldsymbol{p}=\boldsymbol{\eta}$; that is, $\widehat{v}_{\boldsymbol{p}, \boldsymbol{\eta}}^{U}=\mu_{\boldsymbol{p}}$ and $\widehat{v}_{\boldsymbol{\eta}, \boldsymbol{w}}^{U}=\mu_{|\boldsymbol{w}|}$ for any $U \in \widetilde{\mathcal{U}}^{1 / n}$. Moreover, it is worth noting that, when $n=2$, any Choquet integral with respect to a normalized capacity can be expressed as a SUOWA operator [28]. ${ }^{1}$ A summary of the main properties of SUOWA operators can be found in [31].

The constructions we propose in this paper are based on the notion of pseudo-inverse of a function. Next we recall the definition and main properties of pseudo-inverses of strictly increasing functions (see [32, Corollary 3.3 and Remark 3.4]).

Definition 2: Let $f:[a, b] \longrightarrow[c, d]$ be a strictly increasing function where $[a, b]$ and $[c, d]$ are two closed subintervals of the extended real line $[-\infty, \infty]$. Then the pseudo-inverse of $f, f^{(-1)}:[c, d] \longrightarrow[a, b]$, is defined by

$$
f^{(-1)}(y)=\sup \{x \in[a, b] \mid f(x)<y\} .
$$

Remark 1: Let $f:[a, b] \longrightarrow[c, d]$ be a strictly increasing function where $[a, b]$ and $[c, d]$ are two closed subintervals of $[-\infty, \infty]$. Then

1) $f^{(-1)}$ is nondecreasing and continuous.

\footnotetext{
${ }^{1}$ However, this fact does not have to happen in higher dimensions. For instance, an example showing that WOWA and SUOWA operators are different classes of aggregation operators can be found in [3].
} 
2) If $y \in[c, f(a)]$, then $f^{(-1)}(y)=a$; if $\left.\left.y \in\right] f(b), d\right]$, then $f^{(-1)}(y)=b$.

3) If $f$ is a bijection, $f^{(-1)}=f^{-1}$.

4) $\left.f^{(-1)}\right|_{\operatorname{Ran}(f)}: \operatorname{Ran}(f) \longrightarrow[a, b]$ is also strictly increasing, and

$$
\begin{aligned}
\left.f \circ f^{(-1)}\right|_{\operatorname{Ran}(f)} & =\operatorname{id}_{\operatorname{Ran}(f)}, \\
f^{(-1)} \circ f & =\operatorname{id}_{[a, b]} .
\end{aligned}
$$

\section{UNIMODAL WEIGHTING VECTORS}

The notion of unimodal sequence is well known in the field of sequences of real numbers (see, for instance, [33] and references therein). Recently, this concept has been introduced in the framework of weighting vectors [23].

Definition 3: A weighting vector $\boldsymbol{q}=\left(q_{1}, q_{2}, \ldots, q_{n}\right)$ is unimodal if there exists an index $k$ such that $q_{1} \leq \cdots \leq$ $q_{k-1} \leq q_{k} \geq q_{k+1} \geq \cdots \geq q_{n}$.

The family of unimodal weighting vectors is very interesting because it embrace some of the most outstanding weighting vectors used in the literature: nonincreasing, nondecreasing, centered weighting vectors, etc. (see [23]). Notice also that the dual of a unimodal weighting vector is also unimodal. Throughout the paper $\mathcal{W}_{\mathrm{u}}$ will denote the set of unimodal weighting vectors.

Next we show some technical lemmas about unimodal weighting vectors that will be useful in the following sections. The first one was proved in [23].

Lemma 1: Given $\boldsymbol{w} \in \mathcal{W}_{\mathrm{u}}$, if we define

$$
\begin{aligned}
L_{\boldsymbol{w}} & =\left\{l \in N \mid \sum_{i=1}^{l} w_{i}<\frac{l}{n}\right\}, \\
l_{\boldsymbol{w}} & = \begin{cases}0, & \text { if } L_{\boldsymbol{w}}=\varnothing, \\
\max L_{\boldsymbol{w}}, & \text { otherwise, }\end{cases} \\
L^{\boldsymbol{w}} & =\left\{l \in N \mid \sum_{i=1}^{l} w_{i}>\frac{l}{n}\right\}, \\
l^{\boldsymbol{w}} & = \begin{cases}n+1, & \text { if } L^{\boldsymbol{w}}=\varnothing, \\
\min L^{\boldsymbol{w}}, & \text { otherwise, }\end{cases}
\end{aligned}
$$

then

1) $l_{\boldsymbol{w}}<l^{w}$.

2) If $L_{\boldsymbol{w}} \neq \varnothing$, then $L_{\boldsymbol{w}}=\left\{1, \ldots, l_{\boldsymbol{w}}\right\}$.

3) If $L^{\boldsymbol{w}} \neq \varnothing$, then $L^{\boldsymbol{w}}=\left\{l^{\boldsymbol{w}}, \ldots, n-1\right\}$.

According to the previous lemma, each unimodal weighting vector $\boldsymbol{w}$ has associated two indices, $l_{\boldsymbol{w}}$ and $l^{\boldsymbol{w}}$, so if $l \leq l_{\boldsymbol{w}}$, then the average of the $l$ first components of $\boldsymbol{w}$ is less than $1 / n$; and if $l \geq l^{\boldsymbol{w}}(l \neq n)$, then the average of the $l$ first components of $\boldsymbol{w}$ is greater than $1 / n$.

The next lemma shows some relationships between the indices $l_{\boldsymbol{w}}$ and $l^{\boldsymbol{w}}$ of a unimodal weighting vector and its dual.

Lemma 2: Let $\boldsymbol{w} \in \mathcal{W}_{\mathrm{u}}$. Then

1) $l_{\boldsymbol{w}}=0 \Leftrightarrow l^{\overline{\boldsymbol{w}}}=n+1$.

2) If $l_{\boldsymbol{w}} \neq 0$, then $l_{\boldsymbol{w}}+l^{\overline{\boldsymbol{w}}}=n$.
3) $l^{\boldsymbol{w}}=n+1 \Leftrightarrow l_{\overline{\boldsymbol{w}}}=0$.

4) If $l^{\boldsymbol{w}} \neq n+1$, then $l^{\boldsymbol{w}}+l_{\overline{\boldsymbol{w}}}=n$.

Proof: Let $\boldsymbol{w} \in \mathcal{W}_{\mathrm{u}}$. Notice that $\overline{\boldsymbol{w}} \in \mathcal{W}_{\mathrm{u}}$ and that for any $l \in N$,

$$
1=\sum_{i=1}^{l} w_{i}+\sum_{i=l+1}^{n} w_{i}=\sum_{i=1}^{l} w_{i}+\sum_{i=1}^{n-l} \bar{w}_{i} .
$$

Therefore,

$$
\sum_{i=1}^{l} w_{i}<\frac{l}{n} \Leftrightarrow \sum_{i=1}^{n-l} \bar{w}_{i}>\frac{n-l}{n}
$$

that is, $l \in L_{\boldsymbol{w}} \Leftrightarrow n-l \in L^{\overline{\boldsymbol{w}}}$. Hence,

1) $L_{\boldsymbol{w}}=\varnothing \Leftrightarrow L^{\overline{\boldsymbol{w}}}=\varnothing$; so, $l_{\boldsymbol{w}}=0 \Leftrightarrow l^{\overline{\boldsymbol{w}}}=n+1$.

2) If $L_{\boldsymbol{w}} \neq \varnothing$, then $l_{\boldsymbol{w}} \in L_{\boldsymbol{w}}$. Therefore $n-l_{\boldsymbol{w}} \in L^{\overline{\boldsymbol{w}}}$, and consequently, $l^{\overline{\boldsymbol{w}}} \leq n-l_{\boldsymbol{w}}$. Analogously, since $L^{\overline{\boldsymbol{w}}} \neq \varnothing$, $n-l^{\overline{\boldsymbol{w}}} \in L_{\boldsymbol{w}}$ and, consequently, $n-l^{\overline{\boldsymbol{w}}} \leq l_{\boldsymbol{w}}$. From both inequalities we get $l_{\boldsymbol{w}}+l^{\overline{\boldsymbol{w}}}=n$.

Taken into account that $\overline{\overline{\boldsymbol{w}}}=\boldsymbol{w}$, items 3 and 4 can be proven applying the previous cases to $\overline{\boldsymbol{w}}$.

The last lemma establishes that the sequence formed by the averages of the components of a unimodal weighting vector $\boldsymbol{w}$ is nondecreasing up to the index $l_{\boldsymbol{w}}$.

Lemma 3: Let $\boldsymbol{w} \in \mathcal{W}_{\mathrm{u}}$ and $p, q \in N$ with $p<q \leq l_{\boldsymbol{w}}$. Then

$$
\frac{1}{p} \sum_{i=1}^{p} w_{i} \leq \frac{1}{q} \sum_{i=1}^{q} w_{i}
$$

Proof: Let $\boldsymbol{w} \in \mathcal{W}_{\mathrm{u}}$. Notice that it is sufficient to prove that

$$
\frac{1}{q-1} \sum_{i=1}^{q-1} w_{i} \leq \frac{1}{q} \sum_{i=1}^{q} w_{i} .
$$

for any $q \in N \backslash\{1\}$ with $q \leq l_{\boldsymbol{w}}$. Since we have the following equivalence:

$$
\begin{aligned}
\frac{1}{q-1} \sum_{i=1}^{q-1} w_{i} \leq \frac{1}{q} \sum_{i=1}^{q} w_{i} & \Leftrightarrow q \sum_{i=1}^{q-1} w_{i} \leq(q-1) \sum_{i=1}^{q} w_{i} \\
& \Leftrightarrow \sum_{i=1}^{q-1} w_{i} \leq(q-1) w_{q},
\end{aligned}
$$

we are going to prove this last condition. Since $\boldsymbol{w} \in \mathcal{W}_{\mathrm{u}}$, there exists $k \in N$ such that $w_{1} \leq \cdots \leq w_{k-1} \leq w_{k} \geq w_{k+1} \geq$ $\cdots \geq w_{n}$. We distinguish two cases:

1) If $q \leq k$, then $w_{1} \leq \cdots \leq w_{q-1} \leq w_{q}$, and the condition is clearly satisfied.

2) If $q>k$, then suppose by contradiction that $\sum_{i=1}^{q-1} w_{i}>$ $(q-1) w_{q}$. Since $q \leq l_{\boldsymbol{w}}$, we have

$$
w_{q}<\frac{1}{q-1} \sum_{i=1}^{q-1} w_{i}<\frac{1}{n} .
$$

Moreover, since $w_{q} \geq w_{q+1} \geq \cdots \geq w_{n}$, we have

$$
\sum_{i=q}^{n} w_{i} \leq(n-q+1) w_{q}<\frac{n-q+1}{n} .
$$


Therefore,

$$
1=\sum_{i=1}^{q-1} w_{i}+\sum_{i=q}^{n} w_{i}<\frac{q-1}{n}+\frac{n-q+1}{n}=1,
$$

which is absurd.

The properties of unimodal weighting vectors established in this section will be very useful in the proof of some results shown in the next sections.

\section{FiRST FAMILY OF CAPACITIES}

As we have seen in Section II, semiuninorms are essential in the construction of the capacities used in SUOWA operators. In [34], several continuous semiuninorms were introduced by using ordinal sums of aggregation operators. These semiuninorms are based on the expression $h^{(-1)}(h(x)+h(y)-$ $h(1 / n))$, which we will use in the family of semiuninorms that we will give next. Notice that the following result is straightforward taking into account Remark 1.

Proposition 1: Let $h:[0,1] \longrightarrow[-\infty, \infty]$ be a strictly increasing function with $\{-\infty, \infty\} \nsubseteq \operatorname{Ran}(h) .^{2}$ The mapping $U_{h}^{\max }:[0,1]^{2} \longrightarrow[0,1]$ defined by

$$
U_{h}^{\max }(x, y)= \begin{cases}H(x, y) & \text { if } y \leq 1 / n, \\ \max (x, y) & \text { if } y>1 / n,\end{cases}
$$

where $H(x, y)=h^{(-1)}(h(x)+h(y)-h(1 / n))$, is a semiuninorm, and it is continuous when $h$ is continuous.

It is worth noting that $H(0,0)=0$ and that we only consider strictly increasing functions $h$ because the strictly decreasing functions generate the same semiuninorms.

Remark 2: Let $h:[0,1] \longrightarrow[-\infty, \infty]$ be a strictly decreasing function with $\{-\infty, \infty\} \nsubseteq \mathbb{R a n}(h)$. Then $h_{1}=-h$ is a strictly increasing function with $\{-\infty, \infty\} \nsubseteq \operatorname{Ran}\left(h_{1}\right)$. Moreover, $h_{1}^{(-1)}(x)=h^{(-1)}(-x)$ (see [32, property viii of Remark 3.4]). Therefore,

$$
\begin{aligned}
H_{1}(x, y) & =h_{1}^{(-1)}\left(h_{1}(x)+h_{1}(y)-h_{1}(1 / n)\right) \\
& =h^{(-1)}\left(-h_{1}(x)-h_{1}(y)+h_{1}(1 / n)\right) \\
& =h^{(-1)}(h(x)+h(y)-h(1 / n))=H(x, y) .
\end{aligned}
$$

According to Definition 1, the game $v_{\boldsymbol{p}, \boldsymbol{w}}^{U_{\boldsymbol{w}}^{\max }}$, which, for simplicity, will be denoted by $v_{\boldsymbol{p}, \boldsymbol{w}}^{h}$, is given by

$$
v_{\boldsymbol{p}, \boldsymbol{w}}^{h}(A)= \begin{cases}H_{\boldsymbol{p}, \boldsymbol{w}}(A), & \text { if } \mu_{|\boldsymbol{w}|}(A)<|A| / n, \\ \mu_{\boldsymbol{p}}(A), & \text { if } \mu_{|\boldsymbol{w}|}(A)=|A| / n, \\ \max \left(\mu_{\boldsymbol{p}}(A), \mu_{|\boldsymbol{w}|}(A)\right), & \text { if } \mu_{|\boldsymbol{w}|}(A)>|A| / n,\end{cases}
$$

where $A$ is any nonempty subset of $N$ and

$$
\begin{aligned}
H_{\boldsymbol{p}, \boldsymbol{w}}(A) & =|A| H\left(\frac{\mu_{\boldsymbol{p}}(A)}{|A|}, \frac{\mu_{|\boldsymbol{w}|}(A)}{|A|}\right) \\
& =|A| h^{(-1)}\left(h\left(\frac{\mu_{\boldsymbol{p}}(A)}{|A|}\right)+h\left(\frac{\mu_{|\boldsymbol{w}|}(A)}{|A|}\right)-h(1 / n)\right) .
\end{aligned}
$$

\footnotetext{
${ }^{2}$ This condition is imposed to ensure that the expression $h(x)+h(y)-$ $h(1 / n)$ is well defined.
}

Notice that $v_{\boldsymbol{p}, \boldsymbol{w}}^{h}$ is not, in general, a capacity. For instance, consider $h=$ id. Then, $H(x, y)=\max (x+y-1 / n, 0)$ when $y \leq 1 / n$, and

$$
H_{\boldsymbol{p}, \boldsymbol{w}}(A)=\max \left(\mu_{\boldsymbol{p}}(A)+\mu_{|\boldsymbol{w}|}(A)-\frac{|A|}{n}, 0\right) .
$$

Now, take $n=4, \boldsymbol{p}=(0.4,0.3,0.2,0.1)$, and the unimodal weighting vector $\boldsymbol{w}=(0.1,0.1,0.3,0.5)$. Then

$$
v_{\boldsymbol{p}, \boldsymbol{w}}^{h}(\{1\})=0.25>0.2=v_{\boldsymbol{p}, \boldsymbol{w}}^{h}(\{1,4\}) .
$$

However, we can get capacities if we impose additional conditions on the functions $h$. To be specific, let $\mathcal{H}$ be the set of strictly increasing functions $h:[0,1] \longrightarrow[-\infty, \infty]$ with $\{-\infty, \infty\} \nsubseteq \mathbb{R a n}(h)$ and such that

$$
h^{(-1)}(h(t x)-C) \leq t h^{(-1)}(h(x)-C)
$$

for any $C \in[0, h(1 / n)-h(0)], x \in[0,1]$ and $t>1$ such that $t x \in[0,1]$. As we will see in Theorem 1 , the functions belonging to the set $\mathcal{H}$ allow to obtain capacities when the weighting vector $\boldsymbol{w}$ is unimodal. The next proposition will be used in the proof of that theorem.

Proposition 2: Let $\boldsymbol{w} \in \mathcal{W}_{\mathrm{u}}$ and let $h:[0,1] \longrightarrow[-\infty, \infty]$ be a strictly increasing function with $\{-\infty, \infty\} \nsubseteq \operatorname{Ran}(h)$. Then, for any weighting vector $\boldsymbol{p}$, the following holds:

1) If $1 \leq|A| \leq l_{\boldsymbol{w}}$, then $H_{\boldsymbol{p}, \boldsymbol{w}}(A) \leq \mu_{\boldsymbol{p}}(A)$.

2) If $h \in \mathcal{H}$, then $H_{\boldsymbol{p}, \boldsymbol{w}}(A) \leq H_{\boldsymbol{p}, \boldsymbol{w}}(B)$ for any $A, B \subseteq$ $N$, with $\varnothing \neq A \mp B$ and $|B| \leq l_{\boldsymbol{w}}$.

Proof: Let $\boldsymbol{p}$ and $\boldsymbol{w}$ be two weighting vectors with $\boldsymbol{w} \in$ $\mathcal{W}_{\mathrm{u}}$, and $h \in \mathcal{H}$.

1) If $1 \leq|A| \leq l_{\boldsymbol{w}}$, then $\mu_{|\boldsymbol{w}|}(A) /|A|<1 / n$. Therefore, since $h$ is strictly increasing and $h^{(-1)}$ is nondecreasing we have

$$
\begin{aligned}
& H_{\boldsymbol{p}, \boldsymbol{w}}(A) \\
& =|A| h^{(-1)}\left(h\left(\frac{\mu_{\boldsymbol{p}}(A)}{|A|}\right)+h\left(\frac{\mu_{|\boldsymbol{w}|}(A)}{|A|}\right)-h(1 / n)\right) \\
& \leq|A| h^{(-1)}\left(h\left(\frac{\mu_{\boldsymbol{p}}(A)}{|A|}\right)\right)=\mu_{\boldsymbol{p}}(A) .
\end{aligned}
$$

2) If $\varnothing \neq A \varsubsetneqq B$ and $|B| \leq l_{\boldsymbol{w}}$, then by Lemma 3, we have

$$
\frac{\mu_{|\boldsymbol{w}|}(A)}{|A|} \leq \frac{\mu_{|\boldsymbol{w}|}(B)}{|B|},
$$

and, since $h$ is strictly increasing,

$$
\begin{aligned}
& h\left(\frac{\mu_{\boldsymbol{p}}(A)}{|A|}\right)+h\left(\frac{\mu_{|\boldsymbol{w}|}(A)}{|A|}\right)-h(1 / n) \\
& \leq h\left(\frac{|B|}{|A|} \frac{\mu_{\boldsymbol{p}}(B)}{|B|}\right)+h\left(\frac{\mu_{|\boldsymbol{w}|}(B)}{|B|}\right)-h(1 / n) .
\end{aligned}
$$

Now, since $h^{(-1)}$ is nondecreasing and $h \in \mathcal{H}$, we get

$$
\begin{aligned}
& H_{\boldsymbol{p}, \boldsymbol{w}}(A) \\
& =|A| h^{(-1)}\left(h\left(\frac{\mu_{\boldsymbol{p}}(A)}{|A|}\right)+h\left(\frac{\mu_{|\boldsymbol{w}|}(A)}{|A|}\right)-h(1 / n)\right) \\
& \leq|A| h^{(-1)}\left(h\left(\frac{|B|}{|A|} \frac{\mu_{\boldsymbol{p}}(B)}{|B|}\right)+h\left(\frac{\mu_{|\boldsymbol{w}|}(B)}{|B|}\right)-h(1 / n)\right)
\end{aligned}
$$




$$
\begin{aligned}
& \leq|B| h^{(-1)}\left(h\left(\frac{\mu_{\boldsymbol{p}}(B)}{|B|}\right)+h\left(\frac{\mu_{|\boldsymbol{w}|}(B)}{|B|}\right)-h(1 / n)\right) \\
& =H_{\boldsymbol{p}, \boldsymbol{w}}(B) .
\end{aligned}
$$

Theorem 1: Let $\boldsymbol{w} \in \mathcal{W}_{\mathrm{u}}$ and $h \in \mathcal{H}$. Then, for any weighting vector $\boldsymbol{p}, v_{\boldsymbol{p}, \boldsymbol{w}}^{h}$ is a normalized capacity on $N$ given by

$$
v_{\boldsymbol{p}, \boldsymbol{w}}^{h}(A)= \begin{cases}H_{\boldsymbol{p}, \boldsymbol{w}}(A), & \text { if }|A| \leq l_{\boldsymbol{w}}, \\ \mu_{\boldsymbol{p}}(A), & \text { if } l_{\boldsymbol{w}}<|A|<l^{\boldsymbol{w}}, \\ \max \left(\mu_{\boldsymbol{p}}(A), \mu_{|\boldsymbol{w}|}(A)\right), & \text { if }|A| \geq l^{\boldsymbol{w}},\end{cases}
$$

where $A$ is any nonempty subset of $N$.

Proof: Let $\boldsymbol{p}$ and $\boldsymbol{w}$ be two weighting vectors with $\boldsymbol{w} \in$ $\mathcal{W}_{\mathrm{u}}$, and $h \in \mathcal{H}$. From Definition of $v_{\boldsymbol{p}, \boldsymbol{w}}^{h}$ and Lemma 1 we get expression (2). To prove the monotonicity of $v_{\boldsymbol{p}, \boldsymbol{w}}^{h}$ consider $A \varsubsetneqq B$ with $|A| \geq 1$ and $|B|<n$ (the cases $A=\varnothing$ and $B=N$ are trivial). We distinguish the following cases:

1) If $|B| \leq l_{\boldsymbol{w}}$, then, by the second item of Proposition 2 ,

$$
v_{\boldsymbol{p}, \boldsymbol{w}}^{h}(A)=H_{\boldsymbol{p}, \boldsymbol{w}}(A) \leq H_{\boldsymbol{p}, \boldsymbol{w}}(B)=v_{\boldsymbol{p}, \boldsymbol{w}}^{h}(B) .
$$

2) If $|A| \leq l_{\boldsymbol{w}}$ and $|B|>l_{\boldsymbol{w}}$, then, by the first item of Proposition 2,

$$
v_{\boldsymbol{p}, \boldsymbol{w}}^{h}(A)=H_{\boldsymbol{p}, \boldsymbol{w}}(A) \leq \mu_{\boldsymbol{p}}(A) \leq \mu_{\boldsymbol{p}}(B) \leq v_{\boldsymbol{p}, \boldsymbol{w}}^{h}(B) .
$$

3) If $l_{w}<|A|<l^{w}$, then

$$
v_{\boldsymbol{p}, \boldsymbol{w}}^{h}(A)=\mu_{\boldsymbol{p}}(A) \leq \mu_{\boldsymbol{p}}(B) \leq v_{\boldsymbol{p}, \boldsymbol{w}}^{h}(B) .
$$

4) If $|A| \geq l^{w}$, then

$$
\begin{aligned}
v_{\boldsymbol{p}, \boldsymbol{w}}^{h}(A) & =\max \left(\mu_{\boldsymbol{p}}(A), \mu_{|\boldsymbol{w}|}(A)\right) \\
& \leq \max \left(\mu_{\boldsymbol{p}}(B), \mu_{|\boldsymbol{w}|}(B)\right)=v_{\boldsymbol{p}, \boldsymbol{w}}^{h}(B) .
\end{aligned}
$$

The above theorem guarantees the obtaining of normalized capacities when $\boldsymbol{w}$ is unimodal and $h$ satisfies condition (1). Although this condition is rather technical and unintuitive to use, in Section VI we will give a wide variety of functions fulfilling the condition.

\section{SECOND Family of CAPACITIES}

This family of capacities is inspired by the results shown in [14], where the games obtained with the Crescent Method [13] are expressed as

$$
\xi_{\boldsymbol{p}, \boldsymbol{w}}^{U}(A)= \begin{cases}v_{\boldsymbol{p}, \boldsymbol{w}}^{U}(A) & \text { if } \mu_{|\boldsymbol{w}|}(A) \leq|A| / n, \\ \bar{v}_{\boldsymbol{p}, \overline{\boldsymbol{w}}}^{U}(A) & \text { if } \mu_{|\boldsymbol{w}|}(A)>|A| / n,\end{cases}
$$

where $U$ is any semiuninorm such that $U(x, y)=n x y$ for all $y \leq 1 / n$, and $\bar{v}_{\boldsymbol{p}, \overline{\boldsymbol{w}}}^{U}$ is the dual of the game associated with $\boldsymbol{p}, \overline{\boldsymbol{w}}$ and $U$. Notice that the previous expression can be generalized for any semiuninorm $U \in \widetilde{\mathcal{U}}^{1 / n}$. Hence, given two weighting vectors $\boldsymbol{p}$ and $\boldsymbol{w}$, and $U \in \widetilde{\mathcal{U}}^{1 / n}$, we can define the game $\xi_{\boldsymbol{p}, \boldsymbol{w}}^{U}: 2^{N} \longrightarrow \mathbb{R}$ by

$$
\begin{aligned}
& \xi_{\boldsymbol{p}, \boldsymbol{w}}^{U}(A)= \\
& \begin{cases}|A| U\left(\frac{\mu_{\boldsymbol{p}}(A)}{|A|}, \frac{\mu_{|\boldsymbol{w}|}(A)}{|A|}\right) & \text { if } \mu_{|\boldsymbol{w}|}(A) \leq|A| / n, \\
1-(n-|A|) . & \text { if } \mu_{|\boldsymbol{w}|}(A)>|A| / n, \\
U\left(\frac{1-\mu_{\boldsymbol{p}}(A)}{n-|A|}, \frac{1-\mu_{|\boldsymbol{w}|}(A)}{n-|A|}\right) & \end{cases}
\end{aligned}
$$

if $A \neq \varnothing$, and $\xi_{\boldsymbol{p}, \boldsymbol{w}}^{U}(\varnothing)=0$.

Notice that, in general, the game $\xi_{\boldsymbol{p}, \boldsymbol{w}}^{U}$ is not a capacity, so we need to consider the monotonic cover of the game, $\widehat{\xi}_{\boldsymbol{p}, \boldsymbol{w}}^{U}$. Note also that, by construction, $\widehat{\xi}_{\boldsymbol{p}, \boldsymbol{\eta}}^{U}=\mu_{\boldsymbol{p}}$ and $\widehat{\xi}_{\boldsymbol{\eta}, \boldsymbol{w}}^{U}=$ $\mu_{|\boldsymbol{w}|}$ for any $U \in \widetilde{\mathcal{U}}^{1 / n}$. The Choquet integral with respect to the capacity $\widehat{\xi}_{\boldsymbol{p}, \boldsymbol{w}}^{U}$ will be called the Semi-SUOWA operator associated with $\boldsymbol{p}, \boldsymbol{w}$ and $U$.

We now focus on the semiuninorms $U_{h}^{\max }$. In this case, the games $\xi_{\boldsymbol{p}, \boldsymbol{w}}^{U_{h}^{\max }}$, which, for simplicity, will be denoted by $\xi_{\boldsymbol{p}, \boldsymbol{w}}^{h}$, coincide with $v_{\boldsymbol{p}, \boldsymbol{w}}^{h}$ when $\mu_{|\boldsymbol{w}|}(A) \leq|A| / n$ and are the dual of $v_{\boldsymbol{p}, \overline{\boldsymbol{w}}}^{h}$ when $\mu_{|\boldsymbol{w}|}(A)>|A| / n$; that is,

$$
\xi_{\boldsymbol{p}, \boldsymbol{w}}^{h}(A)= \begin{cases}H_{\boldsymbol{p}, \boldsymbol{w}}(A), & \text { if } \mu_{|\boldsymbol{w}|}(A)<|A| / n, \\ \mu_{\boldsymbol{p}}(A), & \text { if } \mu_{|\boldsymbol{w}|}(A)=|A| / n, \\ 1-H_{\boldsymbol{p}, \overline{\boldsymbol{w}}}\left(A^{\mathrm{c}}\right), & \text { if } \mu_{|\boldsymbol{w}|}(A)>|A| / n .\end{cases}
$$

As in the case of $v_{\boldsymbol{p}, \boldsymbol{w}}^{h}$, the games $\xi_{\boldsymbol{p}, \boldsymbol{w}}^{h}$ are not, in general, capacities (the example used for the games $v_{\boldsymbol{p}, \boldsymbol{w}}^{h}$ in Section IV is also valid for these games). But, as in the games $v_{\boldsymbol{p}, \boldsymbol{w}}^{h}$, we can get capacities when $h \in \mathcal{H}$.

Theorem 2: Let $\boldsymbol{w} \in \mathcal{W}_{\mathrm{u}}$ and $h \in \mathcal{H}$. Then, for any weighting vector $\boldsymbol{p}, \xi_{\boldsymbol{p}, \boldsymbol{w}}^{h}$ is a normalized capacity on $N$ given by

$$
\xi_{\boldsymbol{p}, \boldsymbol{w}}^{h}(A)= \begin{cases}H_{\boldsymbol{p}, \boldsymbol{w}}(A), & \text { if }|A| \leq l_{\boldsymbol{w}}, \\ \mu_{\boldsymbol{p}}(A), & \text { if } l_{\boldsymbol{w}}<|A|<l^{\boldsymbol{w}}, \\ 1-H_{\boldsymbol{p}, \overline{\boldsymbol{w}}}\left(A^{\mathrm{c}}\right), & \text { if }|A| \geq l^{\boldsymbol{w}},\end{cases}
$$

where $A$ is any nonempty subset of $N$.

Proof: Let $\boldsymbol{p}$ and $\boldsymbol{w}$ be two weighting vectors with $\boldsymbol{w} \in$ $\mathcal{W}_{\mathrm{u}}$, and $h \in \mathcal{H}$. From Definition of $\xi_{\boldsymbol{p}, \boldsymbol{w}}^{h}$ and Lemma 1 we get expression (3). To prove the monotonicity of $\xi_{p, w}^{h}$ consider $A \varsubsetneqq B$ with $|A| \geq 1$ and $|B|<n$ (the cases $A=\varnothing$ and $B=N$ are trivial). We distinguish the following cases:

1) If $|B| \leq l_{\boldsymbol{w}}$, then, by the second item of Proposition 2 ,

$$
\xi_{\boldsymbol{p}, \boldsymbol{w}}^{h}(A)=H_{\boldsymbol{p}, \boldsymbol{w}}(A) \leq H_{\boldsymbol{p}, \boldsymbol{w}}(B)=\xi_{\boldsymbol{p}, \boldsymbol{w}}^{h}(B) .
$$

2) If $|A| \leq l_{\boldsymbol{w}}$ and $l_{\boldsymbol{w}}<|B|<l^{\boldsymbol{w}}$, then, by the first item of Proposition 2,

$$
\xi_{\boldsymbol{p}, \boldsymbol{w}}^{h}(A)=H_{\boldsymbol{p}, \boldsymbol{w}}(A) \leq \mu_{\boldsymbol{p}}(A) \leq \mu_{\boldsymbol{p}}(B)=\xi_{\boldsymbol{p}, \boldsymbol{w}}^{h}(B) .
$$

3) If $l_{\boldsymbol{w}}<|A|<|B|<l^{\boldsymbol{w}}$, then

$$
\xi_{\boldsymbol{p}, \boldsymbol{w}}^{h}(A)=\mu_{\boldsymbol{p}}(A) \leq \mu_{\boldsymbol{p}}(B)=\xi_{\boldsymbol{p}, \boldsymbol{w}}^{h}(B) .
$$


4) If $|A| \leq l_{\boldsymbol{w}}$ or $l_{\boldsymbol{w}}<|A|<l^{\boldsymbol{w}}$, and $|B| \geq l^{\boldsymbol{w}}$, then, by Lemma 2,

$$
\left|B^{\mathrm{c}}\right|=n-|B| \leq n-l^{\boldsymbol{w}}=l_{\overline{\boldsymbol{w}}} .
$$

Now, by the first item of Proposition 2,

$$
\begin{aligned}
\xi_{\boldsymbol{p}, \boldsymbol{w}}^{h}(A) & \leq \mu_{\boldsymbol{p}}(A) \leq \mu_{\boldsymbol{p}}(B)=1-\mu_{\boldsymbol{p}}\left(B^{\mathrm{c}}\right) \\
& \leq 1-H_{\boldsymbol{p}, \overline{\boldsymbol{w}}}\left(B^{\mathrm{c}}\right)=\xi_{\boldsymbol{p}, \boldsymbol{w}}^{h}(B) .
\end{aligned}
$$

5) If $|B|>|A| \geq l^{w}$, then, by Lemma 2 ,

$$
\left|B^{\mathrm{c}}\right|<\left|A^{\mathrm{c}}\right| \leq l_{\overline{\boldsymbol{w}}}
$$

Now, by the second item of Proposition 2,

$$
\xi_{\boldsymbol{p}, \boldsymbol{w}}^{h}(A)=1-H_{\boldsymbol{p}, \overline{\boldsymbol{w}}}\left(A^{\mathrm{c}}\right) \leq 1-H_{\boldsymbol{p}, \overline{\boldsymbol{w}}}\left(B^{\mathrm{c}}\right)=\xi_{\boldsymbol{p}, \boldsymbol{w}}^{h}(B) .
$$

It is worth noting that these capacities have an interesting property: the dual capacity of $\xi_{\boldsymbol{p}, \boldsymbol{w}}^{h}$ coincides with the capacity obtained by using the dual of the weighting vector $\boldsymbol{w}$.

Proposition 3: Let $\boldsymbol{w} \in \mathcal{W}_{\mathrm{u}}$ and $h \in \mathcal{H}$. Then $\bar{\xi}_{\boldsymbol{p}, \boldsymbol{w}}^{h}=\xi_{\boldsymbol{p}, \boldsymbol{w}}^{h}$ for any weighting vector $\boldsymbol{p}$.

Proof: Let $A \varsubsetneqq N, A \neq \varnothing$ (the cases $A=N$ and $A=\varnothing$ are trivial). Since $\bar{\xi}_{\boldsymbol{p}, \boldsymbol{w}}^{h}(A)=1-\xi_{\boldsymbol{p}, \boldsymbol{w}}^{h}\left(A^{\mathrm{c}}\right)$, we distinguish three cases:

1) If $\left|A^{\mathrm{c}}\right| \leq l_{\boldsymbol{w}}$, then, by Lemma $2, n-|A| \leq n-l^{\overline{\boldsymbol{w}}}$; that is, $|A| \geq l^{\bar{w}}$. Therefore,

$$
\bar{\xi}_{\boldsymbol{p}, \boldsymbol{w}}^{h}(A)=1-H_{\boldsymbol{p}, \boldsymbol{w}}\left(A^{\mathrm{c}}\right)=\xi_{\boldsymbol{p}, \boldsymbol{w}}^{h}(A) .
$$

2) If $\left|A^{\mathrm{c}}\right| \geq l^{\boldsymbol{w}}$, then, by Lemma $2, n-|A| \geq n-l_{\overline{\boldsymbol{w}}}$; that is, $|A| \leq l_{\overline{\boldsymbol{w}}}$. Therefore,

$$
\bar{\xi}_{\boldsymbol{p}, \boldsymbol{w}}^{h}(A)=1-\left(1-H_{\boldsymbol{p}, \overline{\boldsymbol{w}}}(A)\right)=H_{\boldsymbol{p}, \overline{\boldsymbol{w}}}(A)=\xi_{\boldsymbol{p}, \overline{\boldsymbol{w}}}^{h}(A) .
$$

3) If $l_{\boldsymbol{w}}<\left|A^{\mathrm{c}}\right|<l^{\boldsymbol{w}}$, then, as in the previous cases, it is easy to check that by Lemma 2 we have $l_{\bar{w}}<|A|<l^{\bar{w}}$. Therefore,

$$
\bar{\xi}_{\boldsymbol{p}, \boldsymbol{w}}^{h}(A)=1-\mu_{\boldsymbol{p}}\left(A^{\mathrm{c}}\right)=\mu_{\boldsymbol{p}}(A)=\xi_{\boldsymbol{p}, \overline{\boldsymbol{w}}}^{h}(A) .
$$

As we will see in the next section, the games obtained with the Crescent Method can also be obtained with the procedure given in this section by using a function that belongs to $\mathcal{H}$. Therefore, when $\boldsymbol{w}$ is unimodal, the games obtained with the Crescent Method are normalized capacities.

\section{FAMILIES OF $H$-FUNCTIONS}

As we have seen, the capacities built in the previous sections are based on the functions $H$, which in turn depend on $h$. Hence, the purpose of this section is to give a wide variety of functions $h$, which will allow us to obtain a broad variety of capacities. Firstly, in the following remark we show that vertical translations and vertical stretches/shrinks of functions $h$ preserve the functions $H$.

Remark 3: Let $h:[0,1] \longrightarrow[-\infty, \infty]$ be a strictly increasing function with $\{-\infty, \infty\} \nsubseteq \mathbb{R a n}(h)$. Then:

1) If $k \in \mathbb{R}$, the function $h_{1}(x)=h(x)+k$ is also a strictly increasing function with $\{-\infty, \infty\} \nsubseteq \mathbb{R} \operatorname{Ran}\left(h_{1}\right)$.
Moreover, $h_{1}^{(-1)}(x)=h^{(-1)}(x-k) .^{3}$ Therefore,

$$
\begin{aligned}
H_{1}(x, y) & =h_{1}^{(-1)}\left(h_{1}(x)+h_{1}(y)-h_{1}(1 / n)\right) \\
& =h_{1}^{(-1)}(h(x)+h(y)-h(1 / n)+k) \\
& =h^{(-1)}(h(x)+h(y)-h(1 / n))=H(x, y) .
\end{aligned}
$$

2) If $k>0$, the function $h_{1}(x)=k h(x)$ is also a strictly increasing function with $\{-\infty, \infty\} \nsubseteq \mathbb{R a n}\left(h_{1}\right)$. Moreover, $h_{1}^{(-1)}(x)=h^{(-1)}(x / k) .{ }^{4}$ Therefore,

$$
\begin{aligned}
H_{1}(x, y) & =h_{1}^{(-1)}\left(h_{1}(x)+h_{1}(y)-h_{1}(1 / n)\right) \\
& =h_{1}^{(-1)}(k(h(x)+h(y)-h(1 / n))) \\
& =h^{(-1)}(h(x)+h(y)-h(1 / n))=H(x, y) .
\end{aligned}
$$

Theorems 1 and 2 allow us to obtain families of normalized capacities by means of functions $h$ that belong to $\mathcal{H}$. However, condition (1) is rather technical and unintuitive to use. For this reason, in the following proposition we provide more intuitive conditions that allow us to get functions belonging to $\mathcal{H}$.

Proposition 4: Let $h:[0,1] \longrightarrow[-\infty, h(1)]$ be a strictly increasing and differentiable bijection with $h(1)<\infty$ and such that the function $g(x)=x h^{\prime}(x)$ is nonincreasing on ]0, 1]. Then:

1) $h \in \mathcal{H}$.

2) The function $\widetilde{h}(x)=-\mathrm{e}^{-h(x)}$ belongs to $\mathcal{H}$.

Proof: Let $h$ be a function satisfying the hypotheses of the proposition. Notice that, since $h$ is a bijection, $h^{(-1)}=h^{-1}$.

1) Consider the condition

$$
h^{-1}(h(t x)-C) \leq t h^{-1}(h(x)-C),
$$

where $C \in[0, \infty], x \in[0,1]$ and $t>1$ with $t x \in[0,1]$. Notice that if $x=0$ or $C=\infty$, then

$$
h^{-1}(h(t x)-C)=0=t h^{-1}(h(x)-C) .
$$

If $x>0$ and $C<\infty$, let $y=t x$. Since $h^{-1}$ is strictly positive in the interval $]-\infty, h(1)]$, condition (4) is equivalent to

$$
\frac{x}{h^{-1}(h(x)-C)} \leq \frac{y}{h^{-1}(h(y)-C)}
$$

where $0<x<y \leq 1$ and $C \geq 0$. The above inequality means that, for any $C \geq 0$, the function

$$
f(x)=\frac{x}{h^{-1}(h(x)-C)}
$$

is nondecreasing in $] 0,1]$, which is equivalent to

$$
h^{-1}(h(x)-C)-x \cdot h^{\prime}(x) \cdot\left(h^{-1}\right)^{\prime}(h(x)-C) \geq 0
$$

for any $C \geq 0$ and $x \in] 0,1] .{ }^{5}$ Since

$$
\left(h^{-1}\right)^{\prime}(h(x)-C)=\frac{1}{h^{\prime}\left(h^{-1}(h(x)-C)\right)},
$$

${ }^{3}$ Since $h_{1}(x)=h(x)+k$, we have $x=h^{(-1)}\left(h_{1}(x)-k\right)$. Hence, if $h_{1}(x)=y$, we get $h_{1}^{(-1)}(y)=h^{(-1)}(y-k)$.

${ }^{4}$ Since $h_{1}(x)=k h(x)$, we have $x=h^{(-1)}\left(h_{1}(x) / k\right)$. Hence, if $h_{1}(x)=y$, we get $h_{1}^{(-1)}(y)=h^{(-1)}(y / k)$.

${ }^{5}$ Notice that in $x=1$ we are considering the left derivative of the functions. 
condition (5) is equivalent to

$$
x h^{\prime}(x) \leq h^{-1}(h(x)-C) \cdot h^{\prime}\left(h^{-1}(h(x)-C)\right)
$$

for any $C \geq 0$ and $x \in] 0,1]$, which is satisfied when the function $g(x)=x h^{\prime}(x)$ is nonincreasing in ]0,1].

2) Consider the function $\widetilde{h}:[0,1] \longrightarrow[-\infty, \widetilde{h}(1)]$ defined by $\widetilde{h}(x)=-\mathrm{e}^{-h(x)}$. It is easy to check that $\widetilde{h}$ is a strictly increasing and differentiable bijection with $\widetilde{h}(1)=-\mathrm{e}^{-h(1)}<\infty$. Consider now the function

$$
\widetilde{g}(x)=x \widetilde{h}^{\prime}(x)=x h^{\prime}(x) \mathrm{e}^{-h(x)} .
$$

By hypothesis, the function $g(x)=x h^{\prime}(x)$ is nonincreasing on $] 0,1]$. Moreover, $g(x)$ is positive on $] 0,1]$, and $\mathrm{e}^{-h(x)}$ is positive and nonincreasing on $\left.] 0,1\right]$. Therefore the function $\widetilde{g}(x)$ is nonincreasing on $] 0,1]$ and, by the first item of this proposition, $\widetilde{h} \in \mathcal{H}$.

Notice that when $\widetilde{h}(x)=-\mathrm{e}^{-h(x)}$, the function $\widetilde{h}^{-1}$ : $\left[-\infty,-\mathrm{e}^{-h(1)}\right] \longrightarrow[0,1]$ is defined by

$$
\widetilde{h}^{-1}(x)=h^{-1}(-\log (-x))
$$

and

$$
\widetilde{H}(x, y)=h^{-1}\left(-\log \left(\mathrm{e}^{-h(x)}+\mathrm{e}^{-h(y)}-\mathrm{e}^{-h(1 / n)}\right)\right) .
$$

It is also worth noting that the condition imposed on the function $x h^{\prime}(x)$ is equivalent to the log-convexity ${ }^{6}$ of the function $h^{-1}$.

Proposition 5: Let $h:[0,1] \longrightarrow[-\infty, h(1)]$ be a strictly increasing and differentiable bijection with $h(1)<\infty$. Then $h^{-1}$ is log-convex on $\left.]-\infty, h(1)\right]$ if and only if the function $g(x)=x h^{\prime}(x)$ is nonincreasing on $\left.] 0,1\right]$.

Proof: Let $h$ be a function satisfying the hypotheses of the proposition. $h^{-1}$ is log-convex when $\log h^{-1}$ is convex; or equivalently, when the function $\left(h^{-1}\right)^{\prime} / h^{-1}$ is nondecreasing; that is, when

$$
\frac{\left(h^{-1}\right)^{\prime}(h(x))}{h^{-1}(h(x))} \leq \frac{\left(h^{-1}\right)^{\prime}(h(y))}{h^{-1}(h(y))}
$$

whenever $x<y$. The above expression is equivalent to

$$
\frac{1}{x h^{\prime}(x)} \leq \frac{1}{y h^{\prime}(y)},
$$

and, since $h^{\prime}$ is strictly positive,

$$
y h^{\prime}(y) \leq x h^{\prime}(x)
$$

whenever $x<y$; that is, the function $g(x)=x h^{\prime}(x)$ is nonincreasing.

In the following proposition we establish conditions under which the functions of the form $h(x)=-(f(x))^{-k}$, where $k>0$, belong to $\mathcal{H}$. Obviously, under the same conditions, the functions $\widetilde{h}(x)=-\mathrm{e}^{-h(x)}=-\mathrm{e}^{(f(x))^{-k}}$ also belong to $\mathcal{H}$.

Proposition 6: Let $f:[0,1] \longrightarrow[0, f(1)]$ be a strictly increasing and twice differentiable bijection, and let $k>0$.

\footnotetext{
${ }^{6}$ Recall that a function $f$ is $\log$-convex when $\log f$ is convex (see, for instance, [35]).
}

If

$$
k \geq \frac{f(x)}{f^{\prime}(x)}\left(\frac{1}{x}+\frac{f^{\prime \prime}(x)}{f^{\prime}(x)}\right)-1
$$

for any $x \in] 0,1]$, then

1) The function $h(x)=-(f(x))^{-k}$ belongs to $\mathcal{H}$.

2) The function $\widetilde{h}(x)=-\mathrm{e}^{(f(x))^{-k}}$ belongs to $\mathcal{H}$.

Proof: Let $f$ be a function satisfying the hypotheses of the proposition.

1) It is immediate to verify that the function $h:[0,1] \longrightarrow$ $[-\infty, h(1)]$ defined by $h(x)=-(f(x))^{-k}$ is a strictly increasing and differentiable bijection with $h(1)<\infty$. Consider now the function $g(x)=x h^{\prime}(x)$. It is easy to check that $g^{\prime}(x) \leq 0$ in $\left.] 0,1\right]$ if and only if

$$
f^{\prime}(x)+x f^{\prime \prime}(x)-\frac{(k+1) x\left(f^{\prime}(x)\right)^{2}}{f(x)} \leq 0
$$

for any $x \in] 0,1]$. Therefore, if

$$
\begin{aligned}
k & \geq \frac{f(x)\left(f^{\prime}(x)+x f^{\prime \prime}(x)\right)}{x\left(f^{\prime}(x)\right)^{2}}-1 \\
& =\frac{f(x)}{f^{\prime}(x)}\left(\frac{1}{x}+\frac{f^{\prime \prime}(x)}{f^{\prime}(x)}\right)-1
\end{aligned}
$$

for any $x \in] 0,1]$, the function $g(x)=x h^{\prime}(x)$ is nonincreasing in $] 0,1]$ and, by the first item of Proposition 4, $h \in \mathcal{H}$.

2) It is immediate by the second item of Proposition 4 .

Notice that, under the hypotheses of the previous proposition, the functions $h^{-1}:\left[-\infty,-(f(1))^{-k}\right] \longrightarrow[0,1]$ and $\widetilde{h}^{-1}:\left[-\infty,-\mathrm{e}^{(f(1))^{-k}}\right] \longrightarrow[0,1]$ are defined by

$$
\begin{aligned}
& h^{-1}(x)=f^{-1}\left((-x)^{-1 / k}\right), \\
& \widetilde{h}^{-1}(x)=f^{-1}\left((\log (-x))^{-1 / k}\right),
\end{aligned}
$$

and, therefore,

$$
\begin{aligned}
& H(x, y)=f^{-1}\left(\left((f(x))^{-k}+(f(y))^{-k}-(f(1 / n))^{-k}\right)^{-1 / k}\right), \\
& \widetilde{H}(x, y)= \\
& f^{-1}\left(\left(\log \left(\mathrm{e}^{(f(x))^{-k}}+\mathrm{e}^{(f(y))^{-k}}-\mathrm{e}^{(f(1 / n))^{-k}}\right)\right)^{-1 / k}\right) .
\end{aligned}
$$

Note also that, since $h(0)=\widetilde{h}(0)=-\infty$, we get $H(x, 0)=$ $H(0, y)=\widetilde{H}(x, 0)=\widetilde{H}(0, y)=0$ for any $x, y \in[0,1]$.

Proposition 6 allows us to obtain a wide variety of functions that belong to $\mathcal{H}$. Next, in the following example, we show some of them and the $H$-functions they generate:

\section{Example 1:}

1) Let $f:[0,1] \longrightarrow[0,1]$ the function defined by $f(x)=$ $x$. Expression (6) becomes

$$
k \geq x \frac{1}{x}-1=0 .
$$

Therefore, when $k>0$ the functions

$$
h(x)=-x^{-k}, \quad \widetilde{h}(x)=-\mathrm{e}^{x^{-k}}
$$


belong to $\mathcal{H}$. Moreover, since $f^{-1}(x)=x$ we get

$$
\begin{aligned}
& H(x, y)=\left(x^{-k}+y^{-k}-n^{k}\right)^{-1 / k}, \\
& \widetilde{H}(x, y)=\left(\log \left(\mathrm{e}^{x^{-k}}+\mathrm{e}^{y^{-k}}-\mathrm{e}^{n^{k}}\right)\right)^{-1 / k} .
\end{aligned}
$$

Note that when $k=1$ we have

$$
H(x, y)=\left(x^{-1}+y^{-1}-n\right)^{-1},
$$

which is of special interest because of its relative simplicity.

2) Let $f:[0,1] \longrightarrow[0, \infty]$ the function defined by $f(x)=$ $-1 / \log (x)$. Expression (6) becomes

$$
k \geq-x \log (x)\left(\frac{1}{x}-\frac{1}{x}\left(1+\frac{2}{\log (x)}\right)\right)-1=1 .
$$

Therefore, when $k \geq 1$ the functions

$$
h(x)=-(-\log (x))^{k}, \quad \tilde{h}(x)=-\mathrm{e}^{(-\log (x))^{k}}
$$

belong to $\mathcal{H}$. Moreover, since $f^{-1}(x)=\mathrm{e}^{-1 / x}$ we get

$$
\begin{aligned}
H(x, y) & =\mathrm{e}^{-\left((-\log (x))^{k}+(-\log (y))^{k}-(\log (n))^{k}\right)^{1 / k}} \\
& =\frac{\mathrm{e}^{(\log (n))^{k}}}{\mathrm{e}^{(\log (1 / x))^{k}} \mathrm{e}^{(\log (1 / y))^{k}}} \\
\widetilde{H}(x, y) & =\mathrm{e}^{-\left(\log \left(\mathrm{e}^{(-\log (x))^{k}}+\mathrm{e}^{(-\log (y))^{k}}-\mathrm{e}^{(\log (n))^{k}}\right)\right)^{1 / k}} .
\end{aligned}
$$

Notice that when $k=1$ we get

a) $H(x, y)=n x y$, function that allows us to get the games obtained with the Crescent Method when we consider the family of capacities introduced in Section V (see [14]).

b) $\widetilde{H}(x, y)=\left(x^{-1}+y^{-1}-n\right)^{-1}$, function obtained in the first item through $H(x, y)$ when $k=1$.

3) Let $f:[0,1] \longrightarrow[0, \infty]$ the function defined by $f(x)=$ $x /(1-x)$. Expression (6) becomes

$$
k \geq x(1-x)\left(\frac{1}{x}+\frac{2}{1-x}\right)-1=x .
$$

Therefore, when $k \geq 1$ the functions

$$
\begin{aligned}
& h(x)=-\left(\frac{x}{1-x}\right)^{-k}=-\left(\frac{1}{x}-1\right)^{k}, \\
& \widetilde{h}(x)=-\mathrm{e}^{(1 / x-1)^{k}}
\end{aligned}
$$

belong to $\mathcal{H}$. Since $f^{-1}(x)=(1+1 / x)^{-1}$ we get

$$
\begin{gathered}
H(x, y)=\left(1+\left((1 / x-1)^{k}+(1 / y-1)^{k}\right.\right. \\
\left.\left.-(n-1)^{k}\right)^{1 / k}\right)^{-1}, \\
\widetilde{H}(x, y)=\left(1+\left(\operatorname { l o g } \left(\mathrm{e}^{(1 / x-1)^{k}}+\mathrm{e}^{(1 / y-1)^{k}}\right.\right.\right. \\
\left.\left.\left.-\mathrm{e}^{(n-1)^{k}}\right)\right)^{1 / k}\right)^{-1} .
\end{gathered}
$$

Notice that when $k=1$ we get the functions obtained in the first item (which is expected by Remark 3),

$$
\begin{aligned}
& H(x, y)=\left(x^{-1}+y^{-1}-n\right)^{-1} \\
& \widetilde{H}(x, y)=\left(\log \left(\mathrm{e}^{x^{-1}}+\mathrm{e}^{y^{-1}}-\mathrm{e}^{n}\right)\right)^{-1} .
\end{aligned}
$$

4) Let $f:[0,1] \longrightarrow[0, \sin (1)]$ the function defined by $f(x)=\sin (x)$. Expression (6) becomes

$k \geq \frac{\sin (x)}{\cos (x)}\left(\frac{1}{x}-\frac{\sin (x)}{\cos (x)}\right)-1=\frac{\sin (x)}{x \cos (x)}-\frac{1}{\cos ^{2}(x)}$.

Notice that in $] 0,1]$ the function

$$
g(x)=\frac{\sin (x)}{x \cos (x)}-\frac{1}{\cos ^{2}(x)}
$$

is bounded above by 0 :

$$
\begin{aligned}
g(x) \leq 0 & \Leftrightarrow \frac{\sin (x)}{x \cos (x)} \leq \frac{1}{\cos ^{2}(x)} \Leftrightarrow \frac{\sin (2 x)}{2 x} \leq 1 \\
& \Leftrightarrow \sin (2 x) \leq 2 x,
\end{aligned}
$$

which is true if $x \in[0,1]$. So, when $k>0$ the functions

$$
h(x)=-(\sin (x))^{-k}, \quad \widetilde{h}(x)=-\mathrm{e}^{(\sin (x))^{-k}}
$$

belong to $\mathcal{H}$. Since $f^{-1}(x)=\arcsin (x)$ we get

$$
\begin{gathered}
H(x, y)=\arcsin (( \\
(\sin (x))^{-k}+(\sin (y))^{-k} \\
\left.\left.-(\sin (1 / n))^{-k}\right)^{-1 / k}\right), \\
\tilde{H}(x, y)=\arcsin \left(\left(\operatorname { l o g } \left(\mathrm{e}^{(\sin (x))^{-k}}+\mathrm{e}^{(\sin (y))^{-k}}\right.\right.\right. \\
\left.\left.\left.-\mathrm{e}^{(\sin (1 / n))^{-k}}\right)\right)^{-1 / k}\right) .
\end{gathered}
$$

5) Let $f:[0,1] \longrightarrow[0,1-\cos (1)]$ the function defined by $f(x)=1-\cos (x)$. Expression (6) becomes

$$
\begin{aligned}
k & \geq \frac{1-\cos (x)}{\sin (x)}\left(\frac{1}{x}+\frac{\cos (x)}{\sin (x)}\right)-1 \\
& =\frac{1-\cos (x)}{x \sin (x)}-\frac{1}{1+\cos (x)} .
\end{aligned}
$$

Notice that in $] 0,1]$ the previous function, called $g(x)$, is bounded above by 0 :

$$
\begin{aligned}
g(x) \leq 0 & \Leftrightarrow \frac{1-\cos (x)}{x \sin (x)} \leq \frac{1}{1+\cos (x)} \\
& \Leftrightarrow \frac{\sin (x)}{x} \leq 1 \Leftrightarrow \sin (x) \leq x,
\end{aligned}
$$

which is true if $x \in[0,1]$. So, when $k>0$ the functions

$$
h(x)=-(1-\cos (x))^{-k}, \quad \widetilde{h}(x)=-\mathrm{e}^{(1-\cos (x))^{-k}}
$$

belong to $\mathcal{H}$. Since $f^{-1}(x)=\arccos (1-x)$ we get

$$
\begin{aligned}
H(x, y)=\arccos (1- & (1-\cos (x))^{-k}+(1-\cos (y))^{-k} \\
& \left.\left.-(1-\cos (1 / n))^{-k}\right)^{-1 / k}\right)
\end{aligned}
$$




$$
\begin{array}{r}
\widetilde{H}(x, y)=\arccos \left(1-\left(\operatorname { l o g } \left(\mathrm{e}^{(1-\cos (x))^{-k}}+\mathrm{e}^{(1-\cos (y))^{-k}}\right.\right.\right. \\
\left.\left.\left.-\mathrm{e}^{(1-\cos (1 / n))^{-k}}\right)\right)^{-1 / k}\right) .
\end{array}
$$

6) Let $f:[0,1] \longrightarrow[0, \mathrm{e}-1]$ the function defined by $f(x)=\mathrm{e}^{x}-1$. Expression (6) becomes

$$
k \geq \frac{\mathrm{e}^{x}-1}{\mathrm{e}^{x}}\left(\frac{1}{x}+1\right)-1=\frac{\mathrm{e}^{x}-x-1}{x \mathrm{e}^{x}} .
$$

Notice that the function

$$
g(x)=\frac{\mathrm{e}^{x}-x-1}{x \mathrm{e}^{x}}
$$

is strictly increasing in $] 0,1]$, and $g(1)=(\mathrm{e}-2) / \mathrm{e}$. Therefore, when $k \geq(\mathrm{e}-2) / \mathrm{e}$ the functions

$$
h(x)=-\left(\mathrm{e}^{x}-1\right)^{-k}, \quad \widetilde{h}(x)=-\mathrm{e}^{\left(\mathrm{e}^{x}-1\right)^{-k}}
$$

belong to $\mathcal{H}$. Since $f^{-1}(x)=\log (1+x)$ we get

$$
\begin{gathered}
H(x, y)=\log \left(1+\left(\left(\mathrm{e}^{x}-1\right)^{-k}+\left(\mathrm{e}^{y}-1\right)^{-k}\right.\right. \\
\left.\left.-\left(\mathrm{e}^{1 / n}-1\right)^{-k}\right)^{-1 / k}\right), \\
\widetilde{H}(x, y)=\log \left(1+\left(\operatorname { l o g } \left(\mathrm{e}^{\left(\mathrm{e}^{x}-1\right)^{-k}}+\mathrm{e}^{\left(\mathrm{e}^{y}-1\right)^{-k}}\right.\right.\right. \\
\left.\left.\left.-\mathrm{e}^{\left(\mathrm{e}^{1 / n}-1\right)^{-k}}\right)\right)^{-1 / k}\right) .
\end{gathered}
$$

7) Given $a \geq 2$, let $f:[0,1] \longrightarrow[0, a-1]$ the function defined by $f(x)=x(a-x)$. Expression (6) becomes

$$
k \geq \frac{x(a-x)}{a-2 x}\left(\frac{1}{x}-\frac{2}{a-2 x}\right)-1=-\frac{a x}{(a-2 x)^{2}} \geq 0 .
$$

Therefore, when $k>0$ the functions

$$
h(x)=-(x(a-x))^{-k}, \quad \widetilde{h}(x)=-\mathrm{e}^{(x(a-x))^{-k}}
$$

belong to $\mathcal{H}$. Moreover, since

$$
f^{-1}(x)=\frac{a}{2}-\sqrt{\left(\frac{a}{2}\right)^{2}-x}
$$

we get

$$
\begin{gathered}
H(x, y)=\frac{a}{2}-\left(\frac{a^{2}}{4}-\left((x(a-x))^{-k}+(y(a-y))^{-k}\right.\right. \\
\left.\left.-n^{2 k} /(n a-1)^{k}\right)^{-1 / k}\right)^{1 / 2}, \\
\widetilde{H}(x, y)=\frac{a}{2}-\left(\frac{a^{2}}{4}-\left(\operatorname { l o g } \left(\mathrm{e}^{(x(a-x))^{-k}}+\mathrm{e}^{(y(a-y))^{-k}}\right.\right.\right. \\
\left.\left.\left.-\mathrm{e}^{n^{2 k} /(n a-1)^{k}}\right)\right)^{-1 / k}\right)^{1 / 2} .
\end{gathered}
$$

We conclude this section by showing some capacities constructed by means of the functions given in Example 1.

Example 2: Let us consider the weighting vectors $\boldsymbol{p}=$ $(0.45,0.3,0.15,0.1)$ and $\boldsymbol{w}=(0.2,0.25,0.35,0.2)$ (notice that $\boldsymbol{w}$ is unimodal). In Table I we show the values (rounded to three decimals) of the following capacities (we have taken
TABLE I

CAPACITIES CONSTRUCTED THROUGH SOME FUNCTIONS OF EXAMPLE 1.

\begin{tabular}{llllll}
\hline Set & $\xi_{\boldsymbol{p}, \boldsymbol{w}}^{h_{1}}$ & $\xi_{\boldsymbol{p}, \boldsymbol{w}}^{h_{2}}$ & $\xi_{\boldsymbol{p}, \boldsymbol{w}}^{h_{5}}$ & $\xi_{\boldsymbol{p}, \boldsymbol{w}}^{\widetilde{h}_{1}}$ & $v_{\boldsymbol{p}, \boldsymbol{w}}^{h_{1}}$ \\
\hline$\{1\}$ & 0.31 & 0.36 & 0.268 & 0.216 & 0.31 \\
$\{2\}$ & 0.231 & 0.24 & 0.223 & 0.208 & 0.231 \\
$\{3\}$ & 0.13 & 0.12 & 0.137 & 0.148 & 0.13 \\
$\{4\}$ & 0.091 & 0.08 & 0.096 & 0.1 & 0.091 \\
$\{1,2\}$ & 0.643 & 0.675 & 0.607 & 0.526 & 0.643 \\
$\{1,3\}$ & 0.529 & 0.54 & 0.519 & 0.491 & 0.529 \\
$\{1,4\}$ & 0.49 & 0.495 & 0.485 & 0.473 & 0.49 \\
$\{2,3\}$ & 0.409 & 0.405 & 0.413 & 0.421 & 0.409 \\
$\{2,4\}$ & 0.367 & 0.36 & 0.373 & 0.386 & 0.367 \\
$\{3,4\}$ & 0.237 & 0.225 & 0.243 & 0.25 & 0.237 \\
$\{1,2,3\}$ & 0.909 & 0.92 & 0.904 & 0.9 & 0.9 \\
$\{1,2,4\}$ & 0.87 & 0.88 & 0.863 & 0.852 & 0.85 \\
$\{1,3,4\}$ & 0.769 & 0.76 & 0.777 & 0.792 & 0.8 \\
$\{2,3,4\}$ & 0.69 & 0.64 & 0.732 & 0.784 & 0.8 \\
$N$ & 1 & 1 & 1 & 1 & 1 \\
\hline
\end{tabular}

$k=1$ in all cases):

1) $\xi_{\boldsymbol{p}, \boldsymbol{w}}^{h_{1}}, \xi_{\boldsymbol{p}, \boldsymbol{w}}^{h_{2}}, \xi_{\boldsymbol{p}, \boldsymbol{w}}^{h_{5}}$, which correspond to the games of Section V where $h_{1}(x)=-1 / x, h_{2}(x)=\log (x)$, and $h_{5}(x)=-1 /(1-\cos (x))$ (i.e., we consider the functions of items 1, 2 and 5 of Example 1). It is worthy of note that, with the weighting vectors used in this example, the capacities obtained through the functions of items 4 , 6 , and 7 (with $a=2$ ), $\xi_{\boldsymbol{p}, \boldsymbol{w}}^{h_{4}}, \xi_{\boldsymbol{p}, \boldsymbol{w}}^{h_{6}}$, and $\xi_{\boldsymbol{p}, \boldsymbol{w}}^{h_{7}}$, are very similar to $\xi_{\boldsymbol{p}, \boldsymbol{w}}^{h_{1}}$ (note that when $k=1, \xi_{\boldsymbol{p}, \boldsymbol{w}}^{h_{3}}=\xi_{\boldsymbol{p}, \boldsymbol{w}}^{h_{1}}$ ).

2) $\xi_{\boldsymbol{p}, \boldsymbol{w}} \widetilde{h}_{1}$, which corresponds to the games of Section $\mathrm{V}$ where $\widetilde{h}_{1}(x)=-\mathrm{e}^{1 / x}$. It is worth pointing out that the values of the capacities $\xi_{\boldsymbol{p}, \boldsymbol{w}}^{\widetilde{h}_{3}}, \xi_{\boldsymbol{p}, \boldsymbol{w}}^{\widetilde{h}_{4}}$, and $\tilde{\xi}_{\boldsymbol{p}, \boldsymbol{w}}^{\breve{h}_{6}}$ are very similar to those of $\widetilde{\xi}_{\boldsymbol{p}, \boldsymbol{w}}^{\widetilde{h}_{1}}$ while those of the capacities $\widetilde{\xi}_{\boldsymbol{p}, \boldsymbol{w}}$ and $\widetilde{\xi}_{\boldsymbol{p}, \boldsymbol{w}}$ (with $a=2$ ) are slightly different in some subsets (notice also that when $k=1, \xi_{\boldsymbol{p}, \boldsymbol{w}}^{\widetilde{h}_{2}}=\xi_{\boldsymbol{p}, \boldsymbol{w}}^{h_{1}}$ ).

3) $v_{\boldsymbol{p}, \boldsymbol{w}}^{h_{1}}$, which corresponds to the games of Section IV where $h_{1}(x)=-1 / x$. Note that when $|A| \leq l_{\boldsymbol{w}}=2$ we get $v_{\boldsymbol{p}, \boldsymbol{w}}^{h}(A)=\xi_{\boldsymbol{p}, \boldsymbol{w}}^{h}(A)$, and when $|A| \geq l^{\boldsymbol{w}}=3$ we have $v_{\boldsymbol{p}, \boldsymbol{w}}^{h}(A)=\max \left(\mu_{\boldsymbol{p}}(A), \mu_{|\boldsymbol{w}|}(A)\right)=v_{\boldsymbol{p}, \boldsymbol{w}}^{h_{1}}(A)$. So, from Table I we can know the values of $v_{\boldsymbol{p}, \boldsymbol{w}}^{h_{2}}, v_{\boldsymbol{p}, \boldsymbol{w}}^{h_{5}}$, and $v_{\boldsymbol{p}, \boldsymbol{w}}^{\widetilde{h}_{1}}$.

As we can see in Table I, the families of capacities introduced in this paper provide a wide variety of alternatives to decision makers. Hence, when choosing a capacity it seems interesting to take into account the desired values in some indices such as the orness degree or the Shapley values.

\section{CONCLUSION}

In this paper we have introduced two broad families of capacities that allow us to generalize those associated with 
the weighted means and the OWA operators. The Choquet integrals associated with the first family of capacities are SUOWA operators whereas the capacities introduced in the second family are expressed as a two-piecewise function where the first piece coincides with a game associated with a SUOWA operator and the second piece is the dual of a game also obtained in the context of SUOWA operators. For this reason, the Choquet integrals associated with these capacities are called Semi-SUOWA operators.

It is worth noting that the only condition required to obtain both families of capacities is that the weighting vector $\boldsymbol{w}$ is unimodal. However, this requirement is not very demanding, since the families of weighting vectors mainly used in the literature (nonincreasing, nondecreasing, centered, etc.) are unimodal. Moreover, the games obtained with the Crescent Method are specific cases of the family of capacities introduced in Section V, so the Crescent Method allows to obtain normalized capacities when $\boldsymbol{w}$ is unimodal.

\section{ACKNOWLEDGEMENTS}

The author is grateful to three anonymous referees for comments and suggestions that helped improve the paper. I am also very grateful to Francisco Cabo for his helpful comments on log-convex functions. This work is partially supported by the Spanish Ministry of Economy and Competitiveness (Project ECO2016-77900-P) and ERDF.

\section{REFERENCES}

[1] R. R. Yager, "On ordered weighted averaging operators in multicriteria decision making," IEEE Trans. Syst., Man, Cybern., vol. 18, no. 1, pp. 183-190, 1988.

[2] V. Torra, "The weighted OWA operator," Int. J. Intell. Syst., vol. 12, no. 2, pp. 153-166, 1997.

[3] B. Llamazares, "Constructing Choquet integral-based operators that generalize weighted means and OWA operators," Inform. Fusion, vol. 23, pp. 131-138, 2015.

[4] G. Beliakov, "A method of introducing weights into OWA operators and other symmetric functions," in Uncertainty Modeling: Dedicated to Professor Boris Kovalerchuk on his Anniversary, V. Kreinovich, Ed. Cham: Springer, 2017, pp. 37-52.

[5] G. Beliakov, T. Calvo, and P. Fuster-Parra, "Implicit averaging functions," Inform. Sci., vol. 417, pp. 96-112, 2017.

[6] B. Llamazares, "An analysis of some functions that generalizes weighted means and OWA operators," Int. J. Intell. Syst., vol. 28, no. 4, pp. 380 393, 2013.

[7] G. Beliakov, "Comparing apples and oranges: The weighted OWA function," Int. J. Intell. Syst., vol. 33, no. 5, pp. 1089-1108, 2018.

[8] B. Llamazares, "A behavioral analysis of WOWA and SUOWA operators," Int. J. Intell. Syst., vol. 31, no. 8, pp. 827-851, 2016.

[9] J.-L. Marichal, "Tolerant or intolerant character of interacting criteria in aggregation by the Choquet integral," Eur. J. Oper. Res., vol. 155, no. 3, pp. 771-791, 2004.

[10] L. S. Shapley, "A value for $n$-person games," in Contributions to the Theory of Games, H. Kuhn and A. W. Tucker, Eds. Princeton: Princeton University Press, 1953, vol. 2, pp. 307-317.

[11] J.-L. Marichal, " $k$-intolerant capacities and Choquet integrals," Eur. $J$. Oper. Res., vol. 177, no. 3, pp. 1453-1468, 2007.

[12] B. Llamazares, "Closed-form expressions for some indices of SUOWA operators," Inform. Fusion, vol. 41, pp. 80-90, 2018.

[13] L. Jin, R. Mesiar, and R. R. Yager, "Melting probability measure with OWA operator to generate fuzzy measure: the Crescent Method," IEEE Trans. Fuzzy Syst., vol. 27, no. 6, pp. 1309-1316, 2019.

[14] B. Llamazares, "On the relationship between the Crescent Method and SUOWA operators," Submitted.

[15] W. J. Dixon, "Simplified estimation from censored normal samples," Ann. Math. Stat., vol. 31, no. 2, pp. 385-391, 1960.
[16] H. Wainer, "Robust statistics: A survey and some prescriptions," J. Educ. Stat., vol. 1, no. 4, pp. 285-312, 1976

[17] B. Llamazares, "An analysis of Winsorized weighted means," Group Decis. Negot., doi: 10.1007/s10726-019-09623-8.

[18] C. Labreuche, "A formal justification of a simple aggregation function based on criteria and rank weights," in DA2PL'2018, Poznan (Poland), November 22-23 2018.

[19] B. Llamazares, "SUOWA operators: An analysis of their conjunctive/disjunctive character," Fuzzy Sets Syst., vol. 357, pp. 117-134, 2019.

[20] M. Maschler and B. Peleg, "The structure of the kernel of a cooperative game," SIAM J. Appl. Math., vol. 15, no. 3, pp. 569-604, 1967.

[21] M. Maschler, B. Peleg, and L. S. Shapley, "The kernel and bargaining set for convex games," Int. J. Game Theory, vol. 1, no. 1, pp. 73-93, 1971.

[22] H.-W. Liu, "Semi-uninorms and implications on a complete lattice," Fuzzy Sets Syst., vol. 191, pp. 72-82, 2012.

[23] B. Llamazares, "Construction of Choquet integrals through unimodal weighting vectors," Int. J. Intell. Syst., vol. 33, no. 4, pp. 771-790, 2018.

[24] G. Choquet, "Theory of capacities," Ann. Inst. Fourier, vol. 5, pp. 131295, 1953.

[25] D. Denneberg, Non-Additive Measures and Integral. Dordrecht: Kluwer Academic Publisher, 1994.

[26] M. Grabisch, J. Marichal, R. Mesiar, and E. Pap, Aggregation Functions. Cambridge: Cambridge University Press, 2009.

[27] R. Mesiar, S. Borkotokey, L. Jin, and M. Kalina, "Aggregation functions and capacities," Fuzzy Sets Syst., vol. 346, pp. 138-146, 2018.

[28] B. Llamazares, "A study of SUOWA operators in two dimensions," Math. Probl. Eng., vol. 2015, pp. Article ID 271 491, 12 pages, 2015.

[29] R. R. Yager and A. Rybalov, "Uninorm aggregation operators," Fuzzy Sets Syst., vol. 80, no. 1, pp. 111-120, 1996.

[30] M. Mas, S. Massanet, D. Ruiz-Aguilera, and J. Torrens, "A survey on the existing classes of uninorms," J. Intell. Fuzzy Syst., vol. 29, no. 3, pp. 1021-1037, 2015.

[31] B. Llamazares, "SUOWA operators: A review of the state of the art," Int. J. Intell. Syst., vol. 34, no. 5, pp. 790-818, 2019.

[32] E. P. Klement, R. Mesiar, and E. Pap, Triangular Norms, ser. Trends in Logic, Studia Logica Library. Dordrecht: Kluwer Academic Publishers, 2000, vol. 8.

[33] F. Brenti, "Log-concave and unimodal sequences in algebra, combinatorics, and geometry: An update," in Jerusalem Combinatorics '93, ser. Contemporary Mathematics, H. Barcelo and G. Kalai, Eds. Providence RI: American Mathematical Society, 1994, vol. 178, pp. 71-89.

[34] B. Llamazares, "SUOWA operators: Constructing semi-uninorms and analyzing specific cases," Fuzzy Sets Syst., vol. 287, pp. 119-136, 2016.

[35] S. Boyd and L. Vandenberghe, Convex Optimization. Cambridge: Cambridge University Press, 2004.

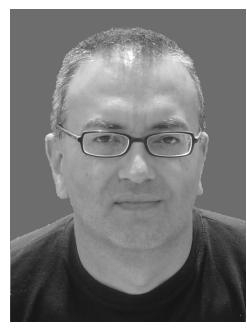

Bonifacio Llamazares received the M.Sc. degree in Mathematics and the Ph.D. degree in Economics, both from the University of Valladolid, Spain, in 1991 and 2000, respectively.

Currently, he is an Associate Professor in the Department of Applied Economics of the University of Valladolid. His domain of research ranges from operations research to fuzzy logic, with special emphasis in the following topics: decision support systems, multicriteria decision making, games, aggregation operators, and fuzzy sets. He is an academic editor of Mathematical Problems in Engineering and a reviewer for numerous journals. He has published papers in several international journals including Computers \& Industrial Engineering, European Journal of Operational Research, Fuzzy Sets and Systems, Group Decision and Negotiation, Information Fusion, Information Sciences, and International Journal of Intelligence Systems. He has participated in the scientific committee of several conferences. 